\title{
éditionsOCDE
}

\section{INSURRECTIONS JIHADISTES EN AFRIQUE DE L'OUEST}

\section{NOTES OUEST-AFRICAINES}

Août 2017 No. 07

Secrétariat du

Club 



\section{INSURRECTIONS JIHADISTES \\ EN AFRIQUE DE L'OUEST : \\ IDÉOLOGIE MONDIALE, CONTEXTE LOCAL, MOTIVATIONS INDIVIDUELLES}

Cette note a été rédigée par

IBRAHIM YAHAYA IBRAHIM

Groupe de recherche sur le Sahel, Université de Floride 


\section{NOTES OUEST-AFRICAINES}

La série Notes ouest-africaines analyse les dynamiques socio-économiques, politiques et sécuritaires que traverse l'Afrique dans une perspective régionale et multidisciplinaire. Elle cherche à stimuler la discussion, rassembler les informations et mieux anticiper les transformations en cours pour les politiques à venir. La série vise à partager des études avec une large audience d'experts, de praticiens du développement, de décisionnaires et de lecteurs avertis. Les Notes sont disponibles en anglais et/ou en français ; les résumés dans les deux langues. Initiées par le Club du Sahel et de l'Afrique de l'Ouest (CSAO) pour éclairer les enjeux ouest-africains, ces analyses sont préparées par son Secrétariat, ses membres et partenaires, les autres départements de l'OCDE, des organisations internationales et autres experts et chercheurs.

En savoir plus sur le Club du Sahel et de l'Afrique de l'Ouest : http://www.oecd.org/fr/csao.

Merci de citer cet ouvrage comme suit:

Ibrahim, I.Y. (2017), ( Insurrections jihadistes en Afrique de l'Ouest : idéologie mondiale, contexte local, motivations individuelles »), Notes ouest-africaines, $\mathrm{N}^{\circ} 07$, Éditions OCDE, Paris. http://dx.doi.org/10.1787/1da64565-fr

Contact auteur : abrayaima@gmail.com

ISSN 2415-1149

Les documents de travail de l'OCDE ne doivent pas être présentés comme exprimant les vues officielles de l'OCDE ou de ses pays membres. Les opinions exprimées et les arguments employés sont ceux des auteurs.

Ce document et toute carte qu'il peut comprendre ne préjugent en rien du statut de tout territoire, de la souveraineté s'exerçant sur ce dernier, du tracé des frontières et limites internationales, et du nom de tout territoire, ville ou région.

Les documents de travail exposent des résultats préliminaires ou des travaux de recherche en cours menés par l'auteur/les auteurs et sont publiés pour stimuler le débat sur un large éventail de questions sur lesquelles l'OCDE travaille. Les commentaires sur les documents de travail sont bienvenus et peuvent être adressés au Club du Sahel et de l'Afrique de l'Ouest, OCDE, 2 rue André-Pascal, 75775 Paris Cedex 16, France.

Autorisé pour publication par Laurent Bossard, Directeur, Secrétariat du Club du Sahel et de l'Afrique de l'Ouest (CSAO/OCDE).

(C) OCDE 2017

Vous êtes autorisés à copier, télécharger ou imprimer du contenu OCDE pour votre utilisation personnelle. Vous pouvez inclure des extraits des publications, des bases de données et produits multimédia de l'OCDE dans vos documents, présentations, blogs, sites Internet et matériel d'enseignement, sous réserve de faire mention de la source OCDE et du copyright. Les demandes pour usage commercial ou de traduction devront être adressées à rights@oecd.org. 


\title{
RÉSUMÉ
}

La récente montée en puissance de mouvements jihadistes en Afrique de l'Ouest, comme Boko Haram dans la région du lac Tchad et Al-Qaïda au Maghreb islamique et ses affiliés dans la région sahélo-saharienne, a surpris les observateurs. La propagation de l'idéologie jihadiste, la facilité de recrutement au sein des populations locales, de même que la capacité à conquérir et administrer des territoires sont des phénomènes sans précédent. La note éclaire sur les facteurs et processus qui contribuent à l'émergence de ces mouvements. Ce travail avance que les insurrections jihadistes en Afrique de l'Ouest découlent de processus à l'œuvre aux niveaux mondial, local et individuel. Au niveau mondial, une idéologie internationale du jihadisme s'est formée et propagée, conceptualisée par des activistes musulmans et des théologiens tenant d'une interprétation particulière de l'islam. Au niveau local, l'appropriation de l'idéologie jihadiste par des ( islamistes ») et son utilisation au service d'un discours qui instrumentalise des revendications sociales et politiques sont un facteur clé. L'affaiblissement des capacités de l'État et les conflits locaux créent des conditions propices à l'émergence de mouvements d'insurrection jihadistes. Au niveau individuel, le processus par lequel des Africains décident de rejoindre des groupes jihadistes relève de motivations idéologiques, stratégiques ou conjoncturelles, favorisées par la détérioration des conditions de vie dans des régions en marge.

Mots clés : jihadisme, terrorisme, islamisme, Sahara-Sahel, Afrique de l'Ouest Classification JEL : D74, F5, H56, N47

\begin{abstract}
À PROPOS DE L'AUTEUR
Ibrahim Yahaya Ibrahim est étudiant en doctorat au Département des sciences politiques de l'Université de Floride et chercheur associé au Groupe de recherche sur le Sahel de cette même université. Sa thèse est consacrée aux contestations politiques et aux discours islamistes dans la région du Sahel, plus particulièrement au Mali, en Mauritanie et au Niger.
\end{abstract}

\section{NOTE AUX LECTEURS}

Cette Note est publiée dans le cadre du partenariat entre l'OCDE et le Groupe de recherche sur le Sahel de l’Université de Floride. La collaboration vise à :

1. Renforcer les liens entre la recherche et les politiques de développement durable qui peuvent aider à mieux anticiper les changements dans la région du Sahel et de l'Afrique de l'Ouest.

2. Promouvoir l'expertise ouest-africaine en renforçant les liens avec les chercheurs et les centres de recherche africains par le biais du réseau du Groupe de recherche sur le Sahel. 



\section{TABLE DES MATIÈRES}

CONTEXTE

LA VAGUE D'INSURRECTION JIHADISTE EN AFRIQUE DE L'OUEST.

JIHADISME : LES FONDEMENTS D’UNE IDÉOLOGIE MONDIALE .8

L'IMPORTANCE DU CONTEXTE LOCAL DANS L'ÉMERGENCE DES « ENTREPRENEURS JIHADISTES"

MOTIVATIONS INDIVIDUELLES DES JIHADISTES PEU GRADÉS 11

PRINCIPALES CONCLUSIONS EN MATIÈRE D'ACTION PUBLIQUE

\section{CONTEXTE}

D epuis une quinzaine années, de nombreuses régions d'Afrique connaissent un développement des mouvements jihadistes, notamment Al-Qaïda au Maghreb islamique (AQMI) dans la région sahélo-saharienne, Boko Haram dans la région du lac Tchad, Al-Shabaab dans la Corne de l'Afrique, Ansar al-Sharia et l'État islamique (EI) dans la région du Maghreb. Ces groupes ont mené de nombreuses attaques à l'origine de dizaines de milliers de victimes et du déplacement de millions de personnes (graphique 1). Entre 2002 et 2017, seize pays africains sont frappés par des attaques jihadistes - prises d'otages, enlèvements de civils, attaques sporadiques de casernements militaires, attentats-suicide dans des églises, des mosquées, des écoles ou des marchés, occupation de territoires, allant parfois jusqu'à vouloir imposer à ces territoires une administration jihadiste-. Des milliers de jeunes Africains issus de milieux sociaux et économiques divers sont piégés par les discours jihadistes. Nombre d'entre eux ont rallié les rangs de groupes qui opèrent sur le continent africain, ou l'EI en Syrie et en Irak (Benmelech et Klor, 2016 : 16). À l'heure où l'EI perd le contrôle de certains de ses bastions au MoyenOrient, il ne fait guère de doutes que certains jihadistes chercheront refuge en Afrique. Autant d'évolutions qui témoignent que le continent africain est devenu l'un des foyers du jihadisme dans le monde.

La vague d'insurrections jihadistes, particulièrement visible en Afrique de l'Ouest, surprend les observateurs. Comment ces régions, dont les sociétés sont réputées pratiquer un islam pacifique, ont-elles donné naissance aux mouvements parmi les plus meurtriers du monde ? Et pourquoi, en dépit des grandes similitudes qui existent entre les pays concernés, le jihadisme s'est-il développé à certains endroits ? Qui sont les jihadistes ouest-africains ? Quel sont leurs profils et leurs motivations ? La note tente de répondre à ces questions en s'intéressant plus particulièrement à AQMI et Boko Haram.

La vague d'insurrections jihadistes en Afrique doit être interprétée comme un enchaînement de processus à l'œuvre à trois niveaux : mondial, local et individuel. Il existe une idéologie jihadiste que certains islamistes et théologiens fondamentalistes ont conceptualisée - et diffusée dans le monde entier - fondée sur une interprétation particulière de l'Islam. Elle est censée répondre aux défis auxquels sont confrontées les sociétés musulmanes contemporaines. Cette idéologie jihadiste est parfois reprise par des islamistes locaux sur le continent africain, pour articuler un discours d'instrumentalisation des doléances des populations locales. Il se veut justifier la violence perpétrée contre l'État et les non-musulmans, et une contribution à la mise en place d'un califat islamique et de la Charia. Un grand nombre d'individus aux milieux sociaux et économiques, et aux motivations variés (religieuses, stratégiques, ou conjoncturelles), ont rejoint ces mouvements. Chaque échelle doit être prise en compte pour tenter de comprendre les mouvements d'insurrections jihadistes en Afrique de l'Ouest. 
Graphique 1

Décès imputables à l'ensemble des groupes (en rouge) et aux groupes islamistes (en vert), 1997-2015

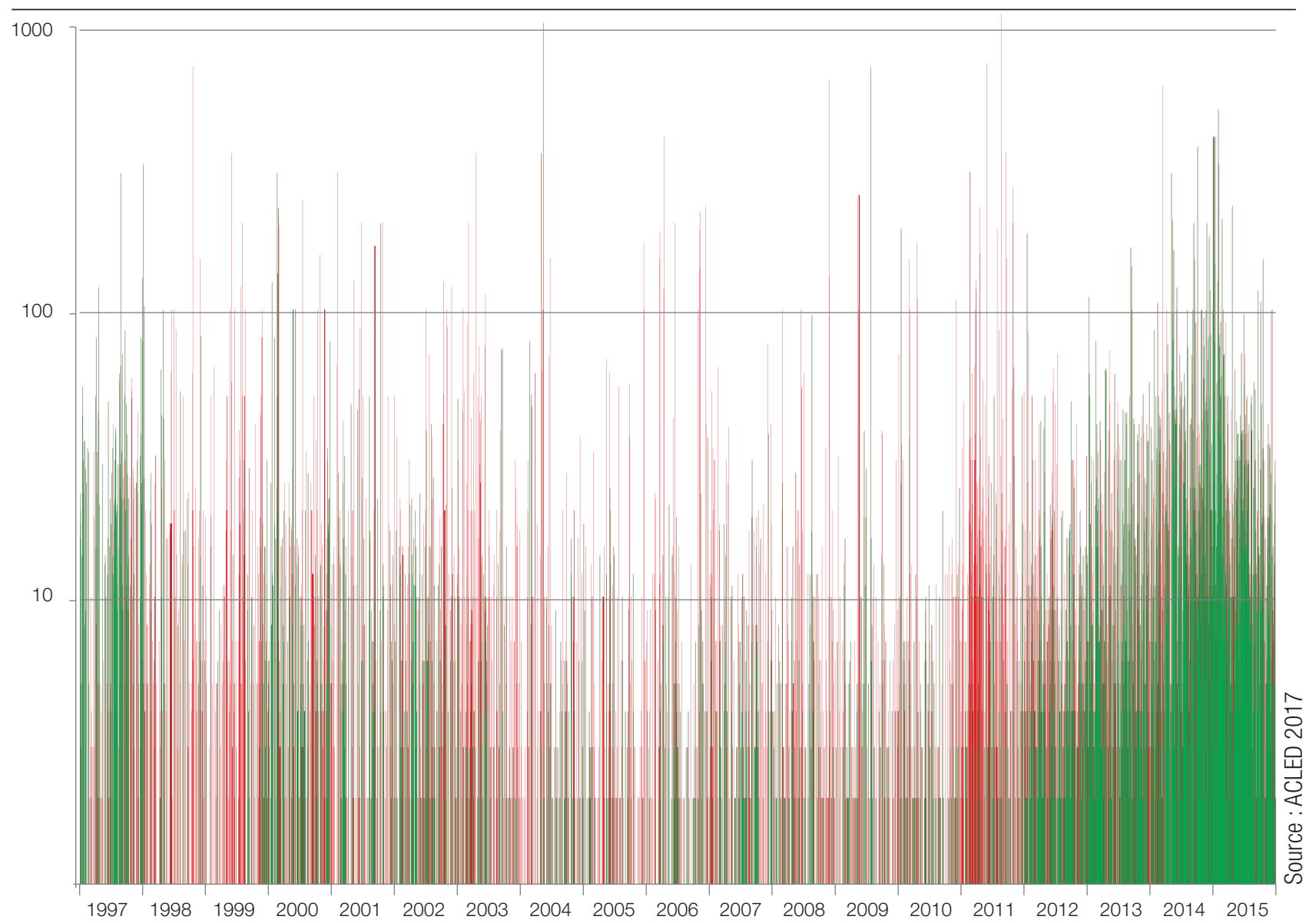

LA VAGUE D'INSURRECTION JIHADISTE EN AFRIQUE DE L'OUEST

I e nouvel épisode d'insurrections jihadistes en Afrique s'est déclenché au début des

L années 2000, lorsque les attentats du 11 septembre et la (" guerre contre le terrorisme ») contribuent à une plus large diffusion de l'idéologie dans le monde. L'émergence des premiers mouvements jihadistes est en outre facilitée par le contexte d'instabilité politique et les conflits civils dans certains pays africains au cours des années 90. De fait, certains mouvements trouvent leur origine dans des conflits préexistants. AQMI est issu de la guerre civile algérienne, déclenchée en 1991 quand un coup de force militaire annule le résultat des premières élections démocratiques, remportées par les islamistes (Abul Ma'ali, 2014). Les islamistes mènent alors une violente campagne contre les dirigeants militaires, destinée pour l'essentiel à faire respecter le résultat du scrutin. Le conflit se poursuit de 1991 à 2002, lorsqu'une branche radicale des insurgés islamistes, le Groupe salafiste pour la prédication et le combat (GSPC), adopte l'idéologie jihadiste d'Al-Qaïda et donne un nouvel objectif à l'insurrection. Il ne s'agit non plus du renversement du pouvoir en Algérie, mais bien de la conduite du jihad dans toute la région sahélo-saharienne. En 2007, le GSPC change de nom pour illustrer ce changement de visée stratégique et l'abandon du conflit national au profit d'un jihad régional.

Toutefois, le jihad n'est pas un phénomène entièrement nouveau en Afrique de l'Ouest. $\mathrm{Au} \mathrm{XIX}^{\mathrm{e}}$ siècle, la région assiste à la montée des mouvements jihadistes peul ou soufi que des cheikhs soufi du groupe ethnique peul mènent contre les royaumes haoussa et bambara (Idrissa, 2009 : 30). Ces premiers conflits jihadistes conduisent à la formation des empires de Sokoto (1804-1903), du Macina (1820-1862) et Toucouleur (1848-1893) (carte 1). 


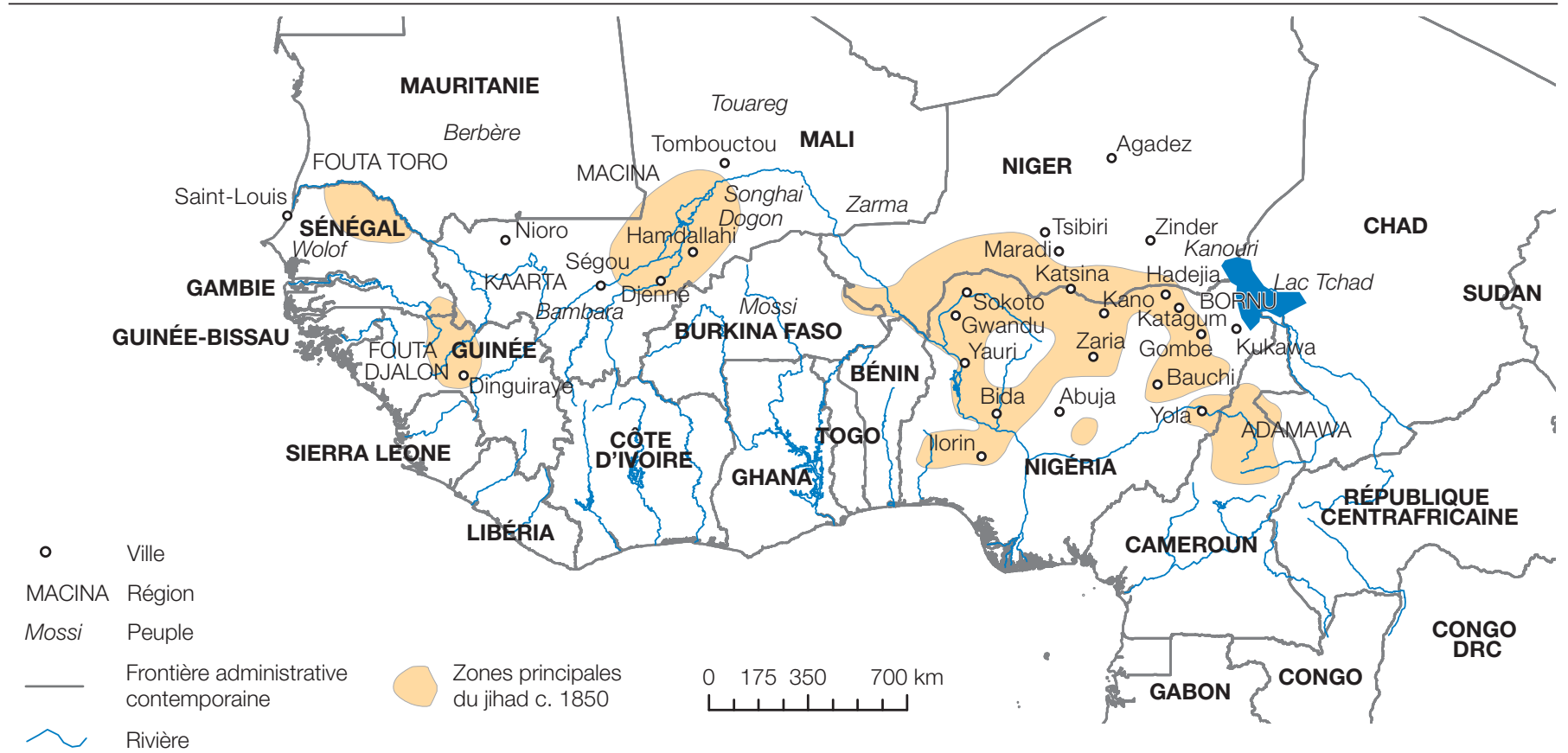

Source : Walther et Miles (à paraître en 2018) ; cartographie : Olivier Walther 2017

Cependant, ces jihads peul du XIX ${ }^{\mathrm{e}}$ siècle étaient différents des insurrections jihadistes contemporaines, dont l'idéologie s'écarte, d’un point de vue épistémologique, du soufisme, reposant sur des motivations sociales plus diverses. L'une des principales caractéristiques du mouvement jihadiste actuel est sa capacité à mobiliser des partisans provenant de milieux sociaux et économiques différents, indépendamment des clivages ethniques, tribaux et raciaux habituels. Cela s'explique en grande partie par le fait que l'islam représente, dans de nombreuses sociétés d'Afrique de l'Ouest, une identité plus englobante, qui dépasse les appartenances ethniques et tribales. Dans leur discours, les (c entrepreneurs du jihad ) en Afrique insistent sur la nécessité pour les musulmans de passer outre ses divisions. À titre d'exemple, invoquant une tradition bien établie lors de précédentes rébellions touareg, Iyad Ag Ghali, le dirigeant d'Ansar Dine, appelle avec insistance à l'union de tous les musulmans du nord du Mali - Touareg, Arabes, Peul, Songhaï et Bambara - pour lutter contre les ( croisés occidentaux ) et leurs alliés locaux (( Liqa'a ma'a Abul fadl ), 2017). Certaines de ses vidéos de propagande montrent des religieux musulmans issus des communautés touareg, arabes et peul qui appellent à l'unité et au jihad contre les infidèles (Imazighen Libya TV, 2013).

Si le fait d'invoquer la religion permet aux jihadistes de mobiliser des partisans issus de différents groupes, les mouvements ne sont pas pour autant homogènes ni unifiés. Ils représentent un attelage hétérogène de groupes divisés selon les clivages qu'ils s'efforcent de surmonter. AQMI connaît des divisions internes motivées par des questions de nationalité ou d'appartenance ethnique. La création du Mouvement pour l'unicité et le jihad en Afrique de l'Ouest (Mujao) résulte ainsi de tensions entre Arabes algériens et maliens pour la direction du mouvement ; les premiers étant accusés par les seconds de monopoliser les postes de commandement au sein d'AQMI et de traiter différemment les jihadistes touareg et arabes. Ces accusations poussent ainsi les Arabes maliens à quitter AQMI pour fonder le Mujao (Abul Ma'ali, 2014). Une autre source de dissensions tient au choix de cibler, ou non, des civils musulmans. Le groupe Jama'atu Ansaril Muslimina fi Biladis Soudan - plus connu sous le nom d'( Ansaru ) - quitte l'organisation Boko Haram en 2012 pour se concentrer sur des cibles internationales, après avoir condamné les atrocités commises contre des civils musulmans (Mohammed, 2014). 
Cependant, en dépit d'importantes divisions internes, ces insurrections jihadistes provoquent des perturbations majeures aux conséquences lourdes. AQMI et Boko Haram réussissent à s'emparer de territoires et à les administrer au moins temporairement. AQMI et des groupes affiliés occupent les deux tiers du territoire malien pendant neuf mois, d'avril 2012 à janvier 2013, tandis que Boko Haram conquiert en 2014 un territoire important dans le nord-est du Nigéria occupé jusqu'en 2016. La capacité des mouvements jihadistes à gouverner les territoires passés sous leur contrôle est toutefois très variable. AQMI et ses groupes affiliés du nord du Mali instituent une administration relativement efficace ainsi qu'un système judiciaire, et tentent d'assurer des services publics (services hospitaliers, distribution d'aide humanitaire, missions de police). La gouvernance exercée par Boko Haram au nord-est du Nigéria se limite quant à elle à une application rigoriste du code pénal respectant la charia.

L'essentiel des ressources des mouvements jihadistes provient habituellement d'impôts, de rançons et de trafics illicites. En Afrique de l'Ouest, les jihadistes perçoivent des millions de dollars en soumettant à l'impôt des entreprises situées dans les zones tombées sous leur contrôle et en prenant part à des activités criminelles - enlèvements, trafics de drogue... AQMI aurait perçu environ 120 millions de dollars US uniquement sous forme de rançons (OCDE, 2013 : 93). De même, Boko Haram acquiert des millions de dollars en imposant les ventes de poissons et de poivres autour du lac Tchad (Oxfam, 2017). Il est souvent avancé que des organisations non gouvernementales (ONG) humanitaires islamiques et d'autres donneurs des pays du Golfe versent des dons aux mouvements jihadistes, mais aucune preuve déterminante n'étaye ces affirmations (Ryder, 2015 : 9).

Les liens pouvant exister entre les mouvements jihadistes africains et les organisations jihadistes mondiales suscitent des inquiétudes, dans la mesure où les mouvements africains ont progressivement fait allégeance à l'EI ou à Al-Qaïda (Siegel, 2017). Si ces rattachements semblent conférer des connexions mondiales aux mouvements jihadistes africains, il convient de ne pas en surestimer l'importance. Une analyse plus poussée montre que ces mouvements ont avant tout un ancrage local, dès lors qu'ils ont surgi sous l'effet d'enjeux sociaux et politiques locaux et qu'ils prétendent en premier lieu satisfaire des revendications locales, et non mondiales. De même, l'hypothèse selon laquelle les mouvements jihadistes africains chercheraient à s'unir et à coordonner leurs actions, inquiète. Or, s'il existe des preuves tangibles de contacts entre AQMI et Boko Haram, sous la forme d'échanges de lettres ou de missions de formation, ces derniers sont demeurés ponctuels. Il n'existe à ce jour aucune preuve que ces deux organisations auraient coordonné et réalisé conjointement des attaques ; à l'inverse, chaque organisation semble concentrer ses actions sur sa propre zone d'influence. Le rôle déterminant dévolu au contexte local n'est donc pas remis en question par de ( prétendus ») liens entre les différents mouvements jihadistes africains.

\section{JIHADISME : LES FONDEMENTS D’UNE IDÉOLOGIE MONDIALE}

es mouvements jihadistes africains partagent des principes idéologiques bien — spécifiques. Premièrement, l'islam est en guerre avec l'Occident et ses alliés dans les sociétés musulmanes, et la lutte armée contre les ennemis de l'islam est un devoir religieux. Deuxièmement, le système reposant sur l'État-nation, les institutions publiques et la démocratie est contraire à l'islam, et doit être remplacé par le modèle du califat régi selon la charia. Troisièmement, un musulman peut être déclaré ( apostat ) ou (( mécréant ) s'il commet des péchés majeurs, auquel cas le recours à la violence à son encontre est légitime. Ces trois concepts forment la quintessence du jihadisme, une idéologie d'ampleur internationale qui motive et justifie les insurrections jihadistes dans le monde entier.

Contrairement aux sectes théologiques que sont le soufisme et le salafisme, qui se limitent essentiellement à l'interprétation de la doctrine religieuse et aux pratiques rituelles, le jihadisme est davantage une idéologie politique qui dit offrir une interprétation cohérente des problématiques sociales et politiques des sociétés musulmanes. En tant que tel, le 
jihadisme est dans la même lignée que le piétisme et l'islamisme. Piétisme, islamisme et jihadisme s'inspirent d'une épistémologie et d'une jurisprudence islamiques propres pour justifier leur vision. Le jihadisme bâtit son idéologie politique et sa conduite extrémiste sur une interprétation essentiellement salafi des textes de l'islam.

Ce sont des activistes musulmans tels Abdallah Azzam (1941-1989), Abu Muhamad Al-Maqdissi (né en 1959) et Oussama Ben Laden (1957-2011) qui les premiers, conceptualisent le jihadisme sur la base de leur interprétation de trois concepts et principes clés. Premièrement, le concept de ( al-wala' wal bara ), littéralement ( la loyauté et le désaveu ») qui, selon l'interprétation jihadiste, stipule qu'un musulman doit une loyauté absolue à tous les autres fidèles et à la communauté des croyants du monde entier, et doit désavouer tous les non-musulmans et leurs systèmes (Thurston, 2016 : 211). Ce principe régit les relations entre les musulmans et les non-musulmans sur la base exclusive des croyances religieuses. En d'autres termes, ( l'amour ) et la ( haine » ne sont déterminés que par l'orientation religieuse de l'autre. Le deuxième concept est celui de la souveraineté, ou (( al-Hakimiyyah », qui explique la vision qu'ont les jihadistes des institutions politiques et de l'autorité (l'Étatnation, la démocratie, les constitutions, etc.). À travers le prisme jihadiste, la souveraineté n'appartient qu'à Dieu. Aucun État ou régime ne peut exercer de souveraineté autrement qu'au nom de Dieu et conformément à la charia (Kepel, 1984 : 48). Les jihadistes rejettent les constitutions des États musulmans modernes au motif de leur non-conformité à ce principe, de même qu'ils rejettent la démocratie qui confère la souveraineté au peuple. Le troisième concept est celui du ( takfir » (excommunication), qui définit les frontières entre les musulmans et les infidèles, et précise les circonstances dans lesquelles un musulman peut être excommunié et avec quelles conséquences (Brigaglia, 2015 : 185-186).

Les idéologues insistent tous sur ces principes dans leurs discours, mais ils ne s'accordent pas sur leur interprétation. Celle du principe d'excommunication (takfir), en particulier, suscite d'importantes controverses, qui ont abouti au schisme récent entre les tenants de l'approche du jihad prônée par al-Qaïda et ceux de la doctrine suivie par l’EI. La première propose une définition étroite du concept de takfir, dont elle limite le champ d'application aux non-musulmans, alors que la deuxième en adopte une acception élargie, et étend son application aux musulmans qui sont en désaccord avec la vision de l'islam propagée par l'EI (Al-Maqlaat, 2016). Le jihad étant exclusivement dirigé contre les non-musulmans, la définition que l'on adopte du concept de takfir influe sur le choix des cibles des violences. Les groupes jihadistes qui ont adopté la conceptualisation de l'EI, en particulier Boko Haram, agissent souvent par des attaques indiscriminées à l'encontre de civils, musulmans ou non. Des groupes tels AQMI, qui se réclament de la vision d'al-Qaïda, ne s'en prennent généralement pas aux civils et ciblent plutôt les intérêts occidentaux et ceux des États.

L'idéologie joue un rôle de premier plan dans l'essor de l'insurrection jihadiste. Elle fournit un prisme à travers lequel les jihadistes voient le monde et réinterprètent les réalités locales. La plupart des revendications des groupes jihadistes ne sont pas nouvelles. Les griefs à l'encontre de l'Occident ont cours depuis longtemps en Afrique de l'Ouest, où ils s'expriment à travers la dénonciation du colonialisme et de l'exploitation illégitime par l'Occident des richesses de cette partie du continent africain. Ils n'ont toutefois que rarement conduit à des actes de violence à l'encontre des Occidentaux. Leur reformulation en termes jihadistes, tels que les (c croisades coloniales ) ou (( pillage ) de la culture et des ressources des musulmans par les « infidèles ), leur confère toutefois une autre portée et justifie la violence à l'encontre de l'Occident et de ses intérêts. L'idéologie redessine les frontières entre les musulmans et les non-musulmans, ou entre les jihadistes et la population, et fournit une justification morale à la violence contre ceux qui n'appartiennent pas à la communauté. Dans la région du lac Tchad les pêcheurs buduma accusent depuis des dizaines d'années les colons haoussa de les priver de leurs ressources économiques. Ces accusations n’ont toutefois jamais dégénéré en (c violence de masse ), en raison notamment du fait que les deux communautés sont musulmanes et que la religion a facilité l'apaisement des tensions entre elles. Toutefois, à mesure que l'idéologie de Boko Haram gagne du terrain dans la région, de nombreux membres des Buduma adoptent l'idéologie 
jihadiste, requalifient les pratiques commerciales des Haoussa en usure - une pratique condamnée par l'islam - et déclarent par conséquent les commerçants haoussa apostats, ce qui en fait des cibles légitimes d'actes de violence. Cette réinterprétation des anciennes récriminations justifie la violence massive perpétrée par Boko Haram à l'encontre des villages haoussa dans la région du lac Tchad (Seignobos, 2016). Enfin, l'idéologie jihadiste sert à inciter les individus à s'engager dans le jihad (Confer plus bas).

\section{L'IMPORTANCE DU CONTEXTE LOCAL DANS L'ÉMERGENCE DES « ENTREPRENEURS JIHADISTES »}

J idéologie est une condition nécessaire à l'essor d'une insurrection jihadiste, mais elle n'est pas suffisante. Il n'existe pas d'insurrection spontanée, sans instigateur ni organisateur. Les instigateurs peaufinent le discours, déclarent que l'insurrection est le seul moyen d'atteindre des objectifs, fournissent les arguments qui apportent une justification morale de la violence et persuadent leurs adeptes de la réussite probable de l'insurrection, en dépit des risques (Sedgwick, 2007 : 16). Pour qu'une insurrection jihadiste éclate, l'idéologie doit être reprise par des activistes musulmans locaux - ou ( (entrepreneurs jihadistes ) - qui formulent alors un discours mieux adapté aux réalités sociales et politiques locales. Le contexte dans lequel ces derniers émergent, ainsi que le processus par lequel ils articulent leur discours et mobilisent leurs disciples, sont déterminants dans la survenue de l'insurrection. Les entrepreneurs jihadistes africains apparaissent généralement dans un contexte d'opportunité politique alimentée par divers facteurs : une capacité limitée de l'État à gouverner, une société profondément divisée, et le recours, par ceux qui remettent en cause l'État, à la violence pour exprimer leurs revendications sociales et politiques. En d'autres termes, les groupes insurgés jihadistes naissent dans un contexte de conflit, qu'ils mettent à profit pour se développer et s'implanter (International Crisis Group, 2016).

La plupart des États africains ne sont ni des États au sens wébérien du terme, avec le monopole de la violence légitime, ni des ( territoires non gouvernés ) ou des « États défaillants ». Ils se caractérisent par un appareil d’État inégal, ce qui signifie que leur monopole de la violence et leur capacité à formuler et mettre en œuvre les politiques ne sont pas uniformes sur l'ensemble de leur territoire (Herbst, 2000 ; Krasner et Risse, 2014). En dépit de la faiblesse de leurs capacités, la plupart d'entre eux disposent d’un « appareil d’État consolidé ») au moins dans la capitale. Ils peuvent s'appuyer sur les capacités militaires et administratives nécessaires pour réglementer la vie en société et résoudre les conflits qui surgissent. La situation est toutefois différente dans les régions rurales et reculées, où leur capacité d'intervention est limitée. Les entrepreneurs jihadistes établissent en général leurs bases dans les régions périphériques, où l’État n'a qu'une capacité d'action restreinte et qui constituent un terrain fertile à l'insurrection. S'ils attaquent parfois des régions où l'État a une forte puissance d'action, ils ne parviennent que rarement à y établir des bases. Dans les rares cas où les mouvements jihadistes implantent des cellules dans les régions métropolitaines -Al Mourabitoune à Nouakchott, en Mauritanie, entre 2008 et 2011, et Boko Haram à Maiduguri, dans le nord-ouest du Nigéria - ces insurrections sont facilement réprimées par l'État. Les jihadistes sont contraints de se retirer dans les régions rurales afin d'échapper à la répression de l’État.

Toutefois, les régions où la capacité d'action étatique est limitée ne présentent pas toutes des conditions propices à l'insurrection. La présence, sur certains territoires, de sociétés profondément divisées offre un terrain plus fertile à l'émergence d'entrepreneurs jihadistes. Les régions africaines où l'État exerce une influence restreinte ne sont pas forcément des espaces « non gouvernés ») (Risse, 2011). Elles sont souvent dirigées par des acteurs non étatiques, notamment des chefs traditionnels, qui maintiennent l'ordre social et font respecter le droit coutumier. Récemment toutefois, l'autorité et la légitimité de ces instances traditionnelles ont reculé sous l'effet des réformes décentralisatrices. De nouveaux acteurs, notamment des élites politiques locales, des responsables religieux et des chefs rebelles, 
émergent et se disputent le contrôle de la vie sociale et politique. Cette concurrence est pacifique dans les régions où la société est relativement homogène et où les règles et normes informelles continuent d'être appliquées, mais là où la société est profondément divisée - selon des clivages religieux ou ethniques - et où les normes informelles sont contestées, la concurrence entre ces acteurs exacerbe les tensions et alimente souvent les conflits. C'est dans ces contextes locaux particuliers que la plupart des entrepreneurs jihadistes se développent.

Ainsi, Mohamed Yusuf (le leader de Boko Haram) est devenu un chef influent dans un contexte d'affaiblissement de l'autorité des dirigeants traditionnels dans le nord du Nigéria et de montée en puissance du sectarisme religieux. S'y conjugue une lutte entre sectes religieuses pour la doctrine, mais également entre les adeptes pour l'accès au pouvoir politique (Last, 2008). Le prestige d'un chef de secte dépend fortement du nombre de ses disciples ; plus ceux-ci sont nombreux, plus il sera courtisé par les dirigeants politiques. Plus son influence politique est importante, plus il gagne de ressources qu'il peut investir pour consolider sa base de fidèles. Selon Murray Last (2008 : 10), Mohamed Yusuf faisait partie de ces chefs de secte en pleine ascension pour qui la nécessité de se démarquer par un discours radical pour attirer l'attention était vitale, et qui, à cette fin, a extrémisé sa doctrine. De même, Iyad ag Ghali est devenu le chef d'Ansar Dine dans le contexte d'un État faible et au sein d'une société divisée selon des clivages ethniques, tribaux et factionnels, avec des autorités informelles et des normes coutumières contestées (Lecocq et Schrijver, 2007 : 156-157). Iyad a lutté, aux côtés de nombreux autres pour le contrôle social et politique du nord du Mali et faisait office d'intermédiaire entre les parties en conflit (Walther et Christopoulos, 2015). Il embrasse l'idéologie jihadiste après l'échec de sa tentative de prendre la tête du Mouvement national de libération de l'Azawad, un mouvement laïc (Bøås, 2015 : 307).

\section{MOTIVATIONS INDIVIDUELLES DES JIHADISTES PEU GRADÉS}

I es raisons qui conduisent un entrepreneur jihadiste à lancer une insurrection peuvent - différer de celles des jihadistes moins gradés. La grande variété des provenances des combattants rend vaine toute tentative de dresser le portrait-robot d'un ( jihadiste type ). Une étude récente consacrée aux motivations des combattants de Boko Haram conclut qu'œ il n'existe pas de portrait démographique des membres de Boko Haram... Certains exerçent un emploi, d’autres pas. Certains ont fréquenté une école laïque, d'autres coranique, ou ont abandonné leur scolarité. Les approches qui s'appuient sur des caractéristiques communes définies ou d'autres facteurs de risque supposés ne semblent pas vouées à réussir ») (Mercy Corps, 2016). Les recrues jihadistes présentent des motivations variées, religieuses, conjoncturelles ou stratégiques, qui ne s'excluent pas mutuellement et coexistent.

Les convictions religieuses jouent un rôle important auprès d'un grand nombre de ceux qui rejoignent des groupes jihadistes. Lors d'un entretien à Gao, un ancien membre du Mujao a expliqué sa décision en ces termes : « Je priais dans la mosquée lorsque les moudjahidin sont entrés dans la ville. Quand je les ai entendus crier « Allahou Akbar, Allahou Akbar, Allahou Akbar ), tout mon corps a commencé à trembler. J'ai senti au plus profond de moi que l'heure était venue, que l'islam devait gouverner. Le lendemain, je suis allé au quartier général du Mujao et je me suis inscrit à une formation militaire. ») Dans le nord du Mali, les membres du Tablighi djama'at et de la secte Waharidji sont parmi les premiers à rejoindre le jihadisme, cependant que, dans le nord-est du Nigéria, Boko Haram trouve son origine dans le mouvement salafiste Ahl As-Sunna wa Al-Jama'a (Thurston, 2016 : 205-207). Les dirigeants de toutes les grandes sectes, dont le soufisme, le salafisme traditionnel et le Tablighi djama'at, ont rejeté le jihadisme comme étant une idéologie déviante. Néanmoins, les doctrines du salafisme, du Tablighi djama'at et de la secte Waharidji présentent des similitudes avec l'idéologie jihadiste, et certains des membres de ces sectes sont davantage prédisposés à adopter les prédications jihadistes. 
La décision des individus de s'enrôler dans un groupe jihadiste résulte également de facteurs contingents ou conjoncturels. Le contexte conflictuel et sécuritaire peut pousser des communautés en quête de protection à faire allégeance à des mouvements jihadistes. Cela fut notamment le cas des bergers peul dans le nord et le centre du Mali et dans le nord-est du Burkina Faso. En 2012, plusieurs centaines de membres de milices peul ont rallié le Mujao dans le cadre d'une alliance contre leurs rivaux touareg (Sangare, 2016). Les liens de parenté influent également dans l'adhésion aux mouvements jihadistes. Les entrepreneurs jihadistes réussissent souvent à recruter dans un premier temps au sein de leur famille, de leur clan puis de leur groupe ethnique, avant d'élargir leur champ d'action à d'autres réseaux. Les liens familiaux et tribaux facilitent ce recrutement, renforcé par un contexte d'insécurité généralisée. La famille et la tribu offrent alors une protection précieuse contre les communautés rivales. Les individus ont peu d'alternative et doivent rallier le groupe soutenu par leur communauté.

D'autres individus, en particulier ceux qui se livrent à des trafics illicites ou liés à des réseaux criminels, s'enrôlent dans des mouvements jihadistes pour des raisons stratégiques ou dictées par leurs activités. Dans le nord du Mali, des jihadistes ont protégé des trafiquants de drogue et d'autres réseaux criminels, qui les ont aidés à leur tour lors des enlèvements et des attaques terroristes. La presse suggérait récemment que les attaques de janvier 2016 contre un hôtel et un café à Ouagadougou auraient été (c sous-traitées )) par le groupe jihadiste Al-Mourabitoune au réseau criminel dirigé par Mimi Ould Baba Ould Cheikh, opérant dans la région de Gao, pour un montant de 10 millions de FCFA (17 000 dollars US) (Le Cam, 2017).

D'autres personnes rejoignent un mouvement jihadiste pour des raisons financières (salaire, butins de pillages) ou, s'agissant des jeunes hommes qui n'ont pas les moyens de se marier, dans la perspective d'épouser une femme enlevée ou une jihadiste (Matfess, 2016).

Enfin, les mesures antiterroristes prises par les gouvernements pour lutter contre les insurrections jihadistes ont souvent nourri les mécontentements et augmenté le nombre de recrutements par les groupes jihadistes. Afin de priver Boko Haram de ressources financières, le Niger, le Nigéria et le Tchad ont ainsi interdit la vente de poivres et de poissons dans la région du lac Tchad, où elle représente les deux principales sources de revenus (Oxfam, 2017).

\section{PRINCIPALES CONCLUSIONS EN MATIÈRE D'ACTION PUBLIQUE}

J insurrection jihadiste en Afrique de l'Ouest est souvent considérée comme un ( phénomène étranger ) organisé et dirigé par des acteurs extérieurs, ou fondé sur une (c idéologie étrangère ») (Holder, 2009 ; Sambe, 2014 : 11). Ce point de vue repose sur la distinction entre un ( islam africain », en référence à la tradition soufie qui a dominé la sphère islamique en Afrique jusqu'au début des années 90, et un ( islam en Afrique ), qui désigne des tendances réformistes de l'islam - en particulier le salafisme - souvent considérées comme ( importées ) du Moyen-Orient (Westerlund et Rosander, 1998). Selon cette analyse, les violences perpétrées au nom de l'islam auraient été quasiment absentes du continent africain avant 1990 dans la mesure où le soufisme permettrait un syncrétisme des croyances islamiques et des traditions africaines, et insisterait sur la promotion de la piété et de la spiritualité sans entrer dans le champ politique. La montée du salafisme observée depuis le début des années 90 aurait donc servi de base à la montée de l'insurrection jihadiste étant donné que, contrairement au soufisme, le salafisme impose de (c désafricaniser » l'islam et de lui adjoindre une dimension politique. 
La vision selon laquelle le soufisme serait pacifique et apolitique est cependant remise en cause par la présence de jihadistes soufis en Afrique de l'Ouest au XIX ${ }^{\mathrm{e}}$ siècle. En outre, ni le soufisme ni le salafisme ne sauraient être réduits à la catégorie de doctrines ( ( locales ) ou (c étrangères ). Qu'ils soient soufis ou salafistes, les musulmans ouest-africains appartiennent à la communauté musulmane mondiale et la dynamique de l'islam dans cette région dépend de la dynamique d'ensemble de cette religion à travers le globe. Influencés par les idéologies islamiques du monde entier, les activistes musulmans d'Afrique de l'Ouest influencent en retour ces idéologies. Il est donc important de souligner l'absence d'une quelconque (c exception africaine ») pour ce qui concerne l'apparition des mouvements d'insurrection jihadiste. Les processus à l'origine de ces mouvements dans la région sont similaires à ceux en cause dans d'autres régions du monde. C'est ainsi une conjonction de facteurs favorables survenant à l'échelon international, local et individuel qui expliquent la vague d'insurrections jihadistes en Afrique de l'Ouest.

La définition des concepts constitue l'un des défis majeurs pour appréhender les mouvements de rébellion jihadistes, dans cette région comme ailleurs. Comment définir le jihadisme selon une vision suffisamment générale pour rendre compte du rôle que jouent certaines interprétations de l'islam, tout en restant assez précis pour éviter d'associer toute la religion islamique à la violence jihadiste ? Il convient tout d'abord de distinguer théologie et idéologie politique. La théologie désigne une simple interprétation des croyances et des dogmes religieux, quand l'idéologie renvoie à une doctrine relative à des choix et comportements politiques. Le jihadisme est une idéologie politique, alors que le soufisme et le salafisme sont des doctrines théologiques. À ce titre, le jihadisme ne doit pas être confondu avec le salafisme. De fait, conférer davantage d'influence à des salafistes traditionnels qui jouissent d'une grande légitimité religieuse peut s'avérer une stratégie payante pour combattre les jihadistes et contenir les extrémismes. Les amalgames opérés entre ces différentes doctrines ont souvent placé des salafistes modérés sous l'emprise des jihadistes, comme ce fut le cas en 2003 en Mauritanie. La diffusion des points de vue du soufisme et du salafisme traditionnels s'est révélée une stratégie très efficace pour isoler les jihadistes dans ce même pays.

Le jihadisme est certes une idéologie mondiale, mais qui se manifeste sous la forme d'insurrections politisées et violentes dans des régions où l'autorité de l'État est peu affirmée et où la société présente des fractures locales que les institutions existantes ne parviennent pas à réduire. Les autorités traditionnelles et le droit coutumier ont joué un rôle important dans le maintien de l'ordre au sein de régions dans lesquelles l'État était peu présent. Toute réforme de décentralisation devrait tenir compte de l'importance de ces institutions informelles et s'attacher à considérer les sources d'autorité et de contrôle social existantes. Il est en effet possible de concevoir des politiques judicieuses qui s'appuient sur les institutions non étatiques afin d'en faire le prolongement utile des institutions officielles. La lutte contre le jihadisme suppose une stratégie inscrite dans la durée et fondée sur la nécessaire mise en place d'une autorité publique efficace et d'institutions légitimes.

Tous les jihadistes ne sont pas des idéologues. Certains, voire la plupart d'entre eux, ont rallié des mouvements jihadistes pour des raisons conjoncturelles ou stratégiques. Identifier la spécificité des griefs et motivations des différents groupes est nécessaire pour bloquer l'appui local des entrepreneurs jihadistes et les recrutements. La négociation peut se révéler possible avec certains jihadistes et ne doit pas être exclue d'emblée. Par ailleurs, les États et les intervenants extérieurs doivent agir avec une extrême prudence, faute de quoi ils pourraient anéantir les efforts des acteurs de terrain les plus efficaces dans la lutte contre le jihadisme. Pour éviter de fournir le moindre argument aux partisans du jihad, ils veilleront à ne jamais donner l'impression de cautionner les prises de position idéologiques allant dans le sens d’une (c croisade ) anti-islamique. 


\section{RÉFÉRENCES}

Abul Ma'ali, M.M. (2014), Al-Qa'idah wa Hulafa'uha fi Azawad, Aljazeera Center for Studies, Doha.

ACLED (2017), « Africa Data, Version 6, 1997-2015 », Armed Conflict Location \& Event Data Project, Université du Sussex, www.acleddata.com/data.

Al-Maqlaat (2016), « The rampant eruption - Mass Takfir by Abu Maysarah Al-Shaami », 16 mai, https://www.justpaste.it/Mass_Takfir (consulté le 13 avril 2017).

Benmelech, E. et E. Klor (2016), "What Explains the Flow of Foreign Fighters to ISIS? », National Bureau of Economic Research, document de travail n²2190, avril, DOI : http://dx.doi. org/10.3386/w22190.

Bøås, M. (2015), "Crime, Coping, and Resistance in the Mali-Sahel Periphery ", African Security, vol. 8, n 4, pp. 299-319, DOI : http://dx.doi.org/10.1080/19392206.2015.1100506.

Brigaglia, A. (2015), "The Volatility of Salafi Political Theology, the War on Terror and the Genesis of Boko Haram ", Diritto e questioni pubbliche, vol. 15, n² 2, pp. 175-201.

Herbst, J. (2000), States and Power in Africa: Comparative Lessons in Authority and Control, Princeton University Press, Princeton, ISBN : 9780691164144

Holder, G. (2009), L'islam, nouvel espace public en Afrique, Paris, Karthala, ISBN : 9782811102548.

Idrissa, A. (2009), "The Invention of Order: Republic Code and Islamic Law in Niger », thèse de doctorat, Université de Floride.

Imazighen Libya TV (2013), Sheikh lyad ag Ghaly, YouTube video, 14 janvier, https://www.youtube. com/watch?v=iXJXmYFczjl\&t=735s (consulté le 11 avril 2017).

International Crisis Group (2016), «Exploiting Disorder: Al-Qaeda and the Islamic State », Crisis Group Special Report, 14 mars, https://www.crisisgroup.org/global/exploiting-disorder-alqaeda-and-islamic-state (consulté le 12 avril 2017).

Kepel, G. (1984), Muslim Extremism in Egypt: The Prophet and Pharaoh, Presses de l'Université de Californie, Berkeley, ISBN : 978-0520239340.

Krasner, S. et T. Risse (2014), « External Actors, State-Building, and Service Provision in Areas of Limited Statehood: Introduction », Governance: An International Journal of Policy, Administration, and Institutions, vol. 27, n² 4, pp. 545-567, DOI : http://dx.doi.org/10.1111/ gove.12065

Last, M. (2008), «The Pattern of Dissent: Boko Haram in Nigeria 2009 », Annual Review of Islam in Africa, vol. 10, pp. 7-11, http://www.humanities.uct.ac.za/usr/cci/news/Murray_Last.pdf.

Le Cam, M. (2017), "Burkina Faso : le principal commanditaire de l'attentat de Ouagadougou identifié ", Le Monde Afrique, 24 mars, http://www.lemonde.fr.

Lecocq, B. et P. Schrijver (2007), "The War on Terror in a Haze of Dust: Potholes and Pitfalls on the Saharan Front ", Journal of Contemporary African Studies, vol. 25, n 1, pp. 141-166, DOI : http://dx.doi.org/10.1080/02589000601157147.

«Liqa'a ma’a Abul fadl, Iyad ag Ghali » (2017), Al-Masra n 45, 3 avril.

Matfess, H. (2016), "Here's why so many people join Boko Haram, despite its notorious violence », The Washington Post, 26 avril, https://www.washingtonpost.com.

Mercy Corps (2016), « Motivation and Empty Promises: Voice of Former Boko Haram Combatants and Nigerian Youth, ", avril, https://www.mercycorps.org/sites/default/files/Motivations\%20 and\%20Empty\%20Promises_Mercy\%20Corps_Full\%20Report_0.pdf (consulté le 12 avril 2017).

Mohammed, K. (2014), "The Message and Methods of Boko Haram », in Pérouse de Montclos, M.A. (ed.) Boko Haram: Islamism, Politics, Security and the State in Nigeria, pp. 9-32, http://horizon.documentation.ird.fr/exl-doc/pleins_textes/divers15-04/010064362.pdf.

OCDE/CSAO (2013), Conflits liés aux ressources et terrorismes : deux facettes de l'insécurité, Éditions OCDE, Paris, DOI : http://dx.doi.org/10.1787/9789264190306-fr.

Oxfam (2017), « L'or rouge et la pêche dans le bassin du lac Tchad " Note d'information Oxfam, février, https://www.oxfam.org/sites/www.oxfam.org/files/file_attachments/bn-red-gold-fishinglake-chad-010217-fr.pdf (consulté le 11 avril 2017).

Risse, T. (2011), "Governance in Areas of Limited Statehood: Introduction and Overview ", in Risse, T. (ed.) Governance Without a State? Policies and Politics in Areas of Limited Statehood, Presses de l'Université de Columbia, New York, pp. 1-35, http://www.jstor.org/stable/10.7312/ riss15120. 
Ryder, N. (2015), The Financial War on Terrorism: A review of counter-terrorism financing strategies since 2001, Routledge, New York, ISBN : 9781134446971.

Sambe, B. (2016), « Dr. Bakary Sambe: Prevention is vital as West African Countries Battle the Rise of Violent Extremism », http://timbuktu-institute.org/prevention-is-vital-as-west-african-countriesbattle-the-rise-of-violent-esxtremism/ (consulté le 12 avril 2017).

Sambe, B. (2014), « Le Sénégal à l'épreuve de la crise sahélienne : nouvelles dynamiques et reconfiguration du champ islamique ", Groupe de recherche sur le Sahel, document de travail $n^{\circ}$ 9, octobre, http://timbuktu-institute.org/wp-content/uploads/2016/04/Working-paper-SahelResearch-Group.pdf.

Sangare, B. (2016), "Le Centre du Mali : épicentre du djihadisme ? ", Groupe de recherche et d'information sur la paix et la sécurité, Note d'analyse du GRIP, 20 mai, http://www.grip.org/fr/ node/2008 (consulté le 12 juillet 2017).

Sedgwick, M. (2007), " Jihad, Modernity, and Sectarianism », Nova Religio: The Journal of Alternative and Emergent Religions, vol. 11, $n^{\circ}$ 2, pp. 6-27, University of California Press, http://www.jstor.org/stable/10.1525/nr.2007.11.2.6.

Seignobos, C. (2016), "Lac Tchad : Tout comprendre de la stratégie des terroristes de Boko Haram ", Le Monde Afrique, 29 avril, http://www.lemonde.fr/.

Siegel, J. (2017), « ISIS in Africa: Implication from Syria and Iraq », Africa Center for Strategic Studies, 17 mars, http://africacenter.org/spotlight/islamic-state-isis-africa-implications-syriairaq-boko-haram-aqim-shabaab/ (consulté le 11 avril 2017).

Thurston, A. (2016), Salafism in Nigeria: Islam, Preaching, and Politics, Presses de l'Université de Cambridge, Cambridge, ISBN : 9781107157439.

Walther, O. et D. Christopoulos (2015), "Islamic terrorism and the Malian rebellion », Terrorism and Political Violence, vol. 27, n 3, pp. 497-519, http://dx.doi.org/10.1080/09546553.2013.809340.

Walther, O. et W. Miles (dir. pub.) (2018), African Border Disorders. Addressing Transnational Extremist Organizations, Abingdon, Routledge, ISBN : 9781138054684 (à paraître).

Westerlund, D. et E.E. Rosander (1998), African Islam \& and Islam in Africa: Encounter Between Sufis and Islamists, Presses de l'Université de l'Ohio, Athènes, ISBN : 978-0-8214-1214-5. 


\section{DANS LA MÊME COLLECTION :}

Allen, T. et P. Heinrigs (2016), « Les nouvelles opportunités de l'économie alimentaire ouest-africaine », Notes ouest-africaines, $\mathrm{N}^{\circ} 1$, Éditions OCDE, Paris. http://dx.doi.org/10.1787/5jlwjg67125f-fr

Lewis, K. et C. Buontempo (2016), « Climate Impacts in the Sahel and West Africa: The Role of Climate Science in Policy Making »), Notes ouest-africaines, N², Éditions OCDE, Paris. http://dx.doi.org/10.1787/5jlsmktwjcd0-en

Gnisci, D. (2016), « Women's Roles in the West African Food System: Implications and Prospects for Food Security and Resilience »), Notes ouest-africaines, N³3, Éditions OCDE, Paris. http://dx.doi.org/10.1787/5jlpl4mh1hxn-en

Staatz, J. et F. Hollinger (2016), « West African Food Systems and Changing Consumer Demands », Notes ouest-africaines, $N^{\circ} 4$, Éditions OCDE, Paris. http://dx.doi.org/10.1787/b165522b-en

Prieto Curiel, R., P. Heinrigs et I. Heo (2017), “ Cities and Spatial Interactions in West Africa: A Clustering Analysis of the Local Interactions of Urban Agglomerations », Notes ouest-africaines, $N^{\circ} 5$, Éditions OCDE, Paris. http://dx.doi.org/10.1787/57b30601-en

Walther, O. (2017), ( Les réseaux de la coopération transfrontalière en Afrique de l'Ouest », Notes ouest-africaines, Nº6, Éditions OCDE, Paris. http://dx.doi.org/10.1787/b7ad4957-fr

En savoir plus : lia.beyeler@oecd.org 